\title{
Polarization preservation of polarized deuteron beams in the electron ion collider at Brookhaven National Laboratory
}

\author{
H. Huang $\odot$, F. Méot, V. Ptitsyn, V. Ranjbar, and T. Roser \\ Brookhaven National Laboratory, Upton, New York 11973, USA
}

(Received 5 September 2019; accepted 21 January 2020; published 11 February 2020)

\begin{abstract}
The physics program at the future electron ion collider (EIC) calls for polarized neutron beams at high energies. The best neutron carriers are ${ }^{3} \mathrm{He}$ nuclei and deuterons. Both neutron carriers are expected to be utilized by the spin physics program in the EIC. Owing to the small magnetic moment anomaly of a deuteron particle, significantly higher magnetic fields are required for spin rotation, so that full Siberian snakes are not feasible. However, the resonance strengths are weak, and the number of resonances is small. It is possible to deal with individual resonances using conventional methods such as a betatron tune jump and vertical ac dipole for intrinsic depolarizing resonances, and a weak partial snake for imperfection resonances. This study employed a realistic magnet ramp rate and parameters (both transverse and longitudinal emittances, as well as realistic lattices) to show that it is possible to accelerate polarized deuterons beyond $100 \mathrm{GeV} / \mathrm{n}$. This paper summarizes the details of the feasibility of a polarized deuteron beam for the future EIC at Brookhaven National Laboratory.
\end{abstract}

DOI: 10.1103/PhysRevAccelBeams.23.021001

\section{INTRODUCTION}

The collision of polarized proton beams has been an essential component of the Relativistic Heavy Ion Collider (RHIC) operations in the past two decades. To preserve the proton polarization, a pair of Siberian snakes [1] have been used in each of the two rings [2]. The deuteron is on the list of species to be collided in the future electron ion collider (EIC) [3], so that the spin properties of the neutron can be studied relative to those of the proton. The EIC is under design at Brookhaven National Laboratory (BNL), and will utilize one of the existing RHIC rings as an ion ring. As there is no easy method of accelerating polarized neutrons to high energies, a polarized deuteron beam provides an alternative solution. A deuteron consists of a proton and a neutron. One important feature of the deuteron is its very small anomalous magnetic g-factor of $G=-0.1426$. This has a profound effect on the manipulation of its spin in a synchrotron. The Siberian snake scheme employed in RHIC does not work for a polarized deuteron. Owing to the small value of $G$, a considerably higher magnetic field is required for spin rotation. The required magnetic field is so high that Siberian snakes are not feasible. On the other hand, with the small value of $G$ the resonance strengths are

Published by the American Physical Society under the terms of the Creative Commons Attribution 4.0 International license. Further distribution of this work must maintain attribution to the author(s) and the published article's title, journal citation, and DOI. expected to be weaker and the energy separation between resonances is expected to be wider than in the proton case.

In fact, there are similar constraints in medium-energy synchrotrons for polarized protons, such as the Alternating Gradient Synchrotron (AGS). The magnetic field from a full snake is too strong and the associated orbit excursion is too large at low and medium energies. The imperfection resonances are overcome by either harmonic orbit correction (as adopted in the 1980s in the AGS [4] and now in the AGS Booster [5]) or partial snakes (as currently used in the AGS [6]). The intrinsic resonances can be overcome using several approaches. First, the resonance crossing speed can be increased by betatron tune jumps, such as in the AGS in the 1980s, at 0.2 units in one turn [4] or 10 turns for strong resonances [7] and 0.04 units in 37 turns for weak resonances [8]. Second, the strong intrinsic resonances can be enhanced by creating coherent betatron oscillations using an ac dipole [9]. Third, a radial jump can be utilized for weak intrinsic resonances [10]. Fourth, a stronger partial snake can be utilized to generate a sufficiently large spin-tune gap such that the intrinsic resonances can be avoided when the betatron tune is in the spin-tune gap [11]. These ideas have previously been tested in the AGS. Taking advantage of the small number of resonances, one can address particular resonances by applying the known techniques, such as a tune jump, or using a partial Siberian snake.

There have been multiple efforts to study the feasibility of accelerating polarized deuterons in RHIC [12-14]. These studies have shown that the acceleration of a polarized deuteron beam in RHIC accelerator chain is feasible, and several techniques and relevant parameters 
have been presented. Some conclusions of previous studies are summarized as follows: The imperfection resonances can be overcome by a partial snake. However, the estimate of the required partial snake strength has varied among different authors from $0.06 \%$ to $0.5 \%$. Existing Siberian snakes have been proposed for utilization as partial snakes for deuterons by some authors, while others have proposed adding a new solenoid. It has been proposed to overcome intrinsic resonances using a fast vertical tune jump of 0.003 in one turn [12] or an ac dipole [13].

These analyses were mostly conducted before RHIC was operational. There are several motivations for a new study of polarized deuteron acceleration in the EIC at BNL. First, as the realistic RHIC magnet ramp rate is slower than assumed in these studies, the required partial snake strength must be reevaluated. The detector solenoids should be considered in this process. Second, the spin-tune spread effect was not previously considered for either imperfection or intrinsic resonances. Third, a fast one-turn tune jump can easily result in emittance growth, as observed during the experiment in the AGS in the 1980s [4]. A benign tune jump system with a tune jump over 37 turns has been implemented in the AGS [8], which mitigated the emittance growth problem. Owing to the weak resonance strength, such a tune jump system with a slower tune jump is sufficient. As the resonance strengths are weak and vary over a wide range, an ac dipole would not be able to overcome these at a practical strength. This study analyzes the imperfection and intrinsic resonance spectra for BNL EIC lattices. We then estimate the required partial snake strength and tune jump system requirements. The paper is organized as follows. Section II describes the polarized deuteron before injection into the EIC ion ring. Then, Sec. III discusses methods to overcome imperfection resonances, and Sec. IV presents details on how to reach longitudinal polarization in the collision region. Section V discusses methods to overcome intrinsic resonances and Sec. VI discusses the overlapping resonances and synchrotron sidebands. Finally a summary is provided in Sec. VII.

\section{POLARIZATION IN THE INJECTORS}

The AGS Booster energy range [15] for the deuteron is $0.95 \mathrm{GeV}-1.3 \mathrm{GeV}$, with $|G \gamma|$ in the range of 0.14 to 0.2 . There are no imperfection resonances in the Booster. If the fractional tune is not between 0.14 and 0.2 (or 0.8-0.86), then intrinsic spin resonances are also avoided.

The AGS energy range is from $1.3 \mathrm{GeV}$ to $10 \mathrm{GeV}$, with a $|G \gamma|$ range of 0.2 to 1.5 . There is only one imperfection resonance in this range, at $|G \gamma|=1$, and this resonance is very weak. There are three intrinsic resonances, but none of these are enhanced with the superperiod of 12 . Their strengths, calculated from DEPOL [16], are very small. For the given AGS ramp rate, none of these resonances cause a polarization loss.
Because there is a horizontal bending section between two vertical bending sections, there is a spin mismatch issue for protons in the AGS-to-RHIC (AtR) transfer line. The reason for the spin mismatch is as follows: there are two opposite and equal vertical bends interleaved with a horizontal bend. For protons, these yield a series of rotations that do not commute, and end up leaving the spin vector tilted slightly away from the vertical axis. However, with such a small $G$ value for deuterons and no snake in the AGS, the spin rotation angles around both the vertical and horizontal axes are approximately commutable with a small rotation angle. Thus, spin matching in the AtR line is not an issue for the deuteron, and tracking with ZGOUBI [17] confirms this. A comparison between the vertical components for the deuteron and proton in the AtR transfer line is presented in Figs. 1 and 2.

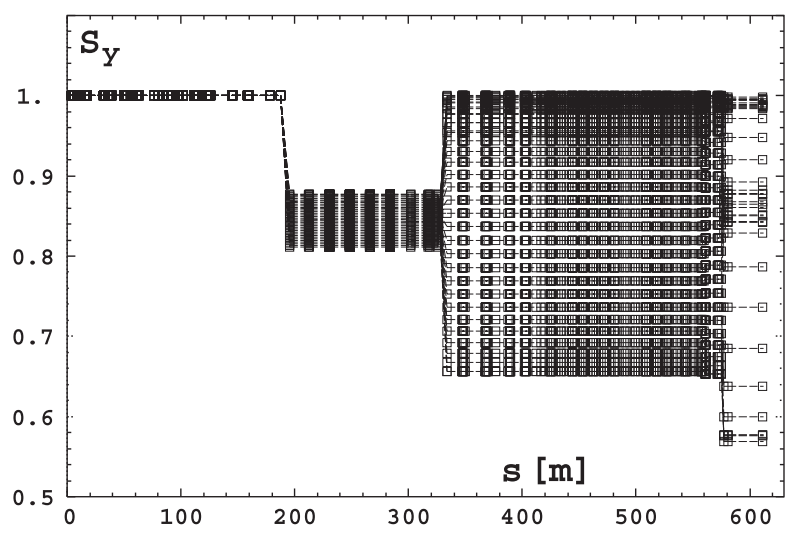

FIG. 1. The ZGOUBI tracking results for the vertical spin components of 36 proton particles with different energies ranging from $21.8 \mathrm{GeV}$ to $29.8 \mathrm{GeV}$ in the AtR beam line. The vertical spin can vary significantly through the AtR line.

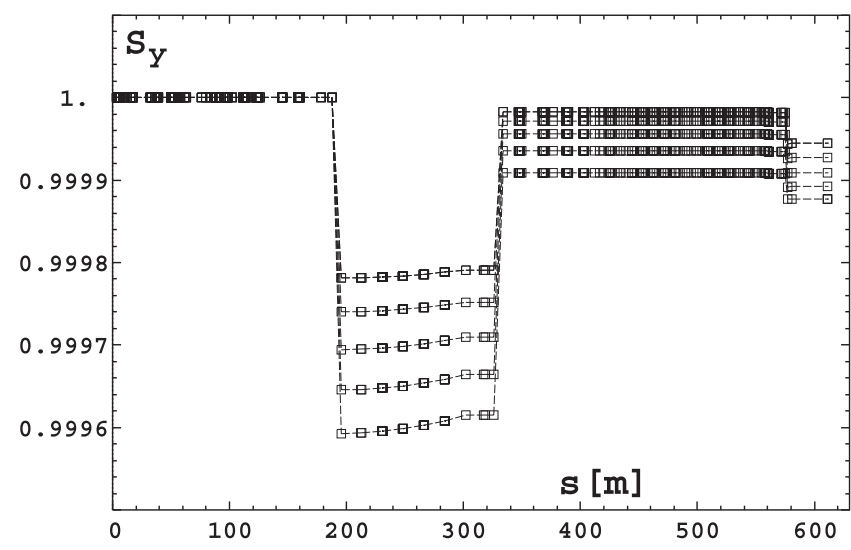

FIG. 2. The ZGOUBI tracking results for the vertical spin components of six deuteron particles with different energies ranging from $10 \mathrm{GeV}$ to $13.1 \mathrm{GeV}$ in the AtR beam line. There is no significant variation in the final vertical spin. 


\section{OVERCOMING IMPERFECTION SPIN RESONANCES}

The challenge of polarization preservation occurs in the ion ring of the BNL EIC, where the deuteron energy range is from $10 \mathrm{GeV}$ to $137.5 \mathrm{GeV}$, or $|G \gamma|$ from 1.5 to 20.9, with total of 19 imperfection resonances. An energy of $137.5 \mathrm{GeV}$ corresponds to $B \rho$ of the proton at an energy of $275 \mathrm{GeV}$. The nominal ramp rate for unpolarized deuteron operation in RHIC is $d \gamma / \mathrm{dt}=90 / 220$, which corresponds to a resonance crossing rate of $\alpha=1.2 \times 10^{-7}$, where $\alpha$ is defined as $\frac{d G \gamma}{d \theta}$ and $\theta$ is the orbit angle. This only represents approximately one fifth of the rate assumed in previous studies. The imperfection resonance strengths for deuterons have been calculated using DEPOL [16] for several standard RHIC lattices with random orbit errors. From beam-beam analysis and previous experience of RHIC operation, three possible vertical tunes are considered as $0.175,0.224$, and 0.673 . With an rms orbit error of $0.3 \mathrm{~mm}$, the strongest resonance strength is less than 0.0015 .

As the resonance strengths are generally weak, a partial snake can overcome these resonances. The required partial snake strength can be estimated from the Froissart-Stora formula [18]. The localized spin rotation by a partial snake with a strength of $\chi$ is $\chi \delta\left(\theta-\theta_{0}\right)$ and the strength of the generated resonance is the Fourier amplitude $\frac{\chi}{2 \pi} e^{i n \theta_{0}}$, for all integers $n$. Here, $\theta_{0}$ is the relative phase of the resonance. In the presence of an imperfection resonance and a partial snake, the Froissart-Stora formula [18] can be rewritten as

$$
\frac{P_{\mathrm{f}}}{P_{\mathrm{i}}}=2 \exp \left(-\frac{\pi}{2 \alpha}\left|\epsilon+\frac{\chi}{2 \pi} e^{i n \theta_{0}}\right|^{2}\right)-1,
$$

where $P_{\mathrm{i}}$ and $P_{\mathrm{f}}$ are the polarization before and after crossing the resonance, respectively; $\epsilon$ is the resonance strength; and $\alpha=\frac{d\left(G_{\gamma}\right)}{d \theta}$ is the resonance crossing rate. A complete spin-flip occurs if

$$
\chi \gg 2 \pi|\epsilon|+\sqrt{8 \pi \alpha} .
$$

Using the strongest resonance strength of 0.0015 and the given resonance crossing speed $\alpha=1.2 \times 10^{-7}$, for a spin flip of over $99 \%$, the partial snake strength $\chi / \pi$ must be greater than 0.00355 . A strength of 0.0045 , or a $0.45 \%$ partial snake, would satisfy this requirement.

The strengths of existing Siberian snakes for the deuteron have been calculated by tracking particles through the four helical modules with various currents. The strongest strength of an existing snake is $0.015 \%$. This is too weak to be utilized for the deuteron. A solenoid can be added to preserve the polarization through these imperfection resonances. A solenoid of a given strength $B_{\mathrm{s}} L$ rotates the spins of particles by an angle

$$
\chi=(1+G) B_{\mathrm{s}} L / B \rho
$$

and produces an integral of resonance strength

$$
\epsilon_{\mathrm{sol}}=\chi / 2 \pi
$$

at all integer values of $G \gamma$. In the above two equations, $B_{\mathrm{s}}$ is the solenoid magnetic field, $L$ is the effective length of the solenoid, and $B \rho$ is the magnetic rigidity of the beam. When the beam energy increases, the corresponding resonance strength decreases for a constant solenoid field. At the energy of the highest imperfection resonance at $|G \gamma|=20$, the required $0.45 \%$ solenoidal partial snake corresponds to a $15-\mathrm{Tm}$ solenoid field.

The current BNL EIC design includes two detectors: detector 1 and detector 2. Both detectors utilize solenoids, and the field strengths are constant for their physics programs. These two detector solenoids impact the deuteron spin, and their effects need to be considered. If their strengths are sufficiently strong, then they can be used as partial snakes for polarized deuterons. Otherwise, an additional solenoid should be added for polarization preservation. The operational scenarios could involve detector 1 alone, or eventually both detectors running. In the current design, the detector 1 solenoid strength is $15 \mathrm{Tm}$ and the detector 2 solenoid strength is $5.6 \mathrm{Tm}$.

The two detector solenoids are separated in the orbit angle by $\pi / 3$. For the two-detector operation scenario, the total partial snake strength can be calculated from the oneturn spin rotation matrix. In this paper, the indices $(1,2,3)$ stand for (radial, longitudinal, vertical) respectively. Considering two partial snakes separated by $1 / m$ of the accelerator ring, with $\chi_{1}$ and $\chi_{2}$ spin-rotation angles, respectively, the one-turn matrix (OTM) is given by

$T=e^{-i \frac{1}{2} G \gamma\left(2 \pi-\frac{2 \pi}{m}-\theta\right) \sigma_{3}} \cdot e^{-i \frac{\chi_{2}}{2} \sigma_{2}} e^{-i \frac{1}{2} G \gamma \frac{2 \pi}{m} \sigma_{3}} e^{-i \frac{\psi_{1}}{2} \sigma_{2}} e^{-i \frac{1}{2} G \gamma \theta \sigma_{3}}$

where $\theta$ is the orbit angle between the observation point and the partial snake with a rotation angle of $\chi_{1}$. Using the Pauli spin matrix relations, the stable spin vector for this OTM can be calculated as

$$
\begin{aligned}
T & =e^{-i \pi \nu_{\mathrm{s}} \hat{\mathrm{c}}_{\mathrm{co}} \cdot \vec{\sigma}} \\
& =I \cos \pi \nu_{\mathrm{s}}-i\left(\sigma_{1} \cos \alpha_{1}+\sigma_{2} \cos \alpha_{2}+\sigma_{3} \cos \alpha_{3}\right) \sin \pi \nu_{\mathrm{s}},
\end{aligned}
$$

where $\nu_{\mathrm{s}}$ is the spin-tune, $\left(\cos \alpha_{1}, \cos \alpha_{2}, \cos \alpha_{3}\right)$ are the stable-spin direction $\hat{n}_{\mathrm{co}}$-directional cosines along the radially outward, longitudinally forward, and vertically up axes, respectively. From the above two equations, the spin-tune with two partial snakes is given by

$$
\begin{aligned}
\cos \pi \nu_{\mathrm{s}}= & \cos \frac{\chi_{1}}{2} \cos \frac{\chi_{2}}{2} \cos G \gamma \pi \\
& -\sin \frac{\chi_{1}}{2} \sin \frac{\chi_{2}}{2} \cos G \gamma\left(\pi-\frac{2 \pi}{m}\right)
\end{aligned}
$$


and the stable-spin directions with two partial snakes are

$$
\begin{aligned}
\cos \alpha_{1}= & \frac{-1}{\sin \pi \nu_{\mathrm{s}}}\left[\cos \frac{\chi_{1}}{2} \sin \frac{\chi_{2}}{2} \sin G \gamma(\pi-\theta)\right. \\
& \left.+\sin \frac{\chi_{1}}{2} \cos \frac{\chi_{2}}{2} \sin G \gamma\left(\pi-\frac{2 \pi}{m}-\theta\right)\right], \\
\cos \alpha_{2}= & \frac{1}{\sin \pi \nu_{\mathrm{s}}}\left[\cos \frac{\chi_{1}}{2} \sin \frac{\chi_{2}}{2} \cos G \gamma(\pi-\theta)\right. \\
& \left.+\sin \frac{\chi_{1}}{2} \cos \frac{\chi_{2}}{2} \cos G \gamma\left(\pi-\frac{2 \pi}{m}-\theta\right)\right], \\
\cos \alpha_{3}= & \frac{1}{\sin \pi \nu_{\mathrm{s}}}\left[\cos \frac{\chi_{1}}{2} \cos \frac{\chi_{2}}{2} \sin G \gamma \pi\right. \\
& \left.-\sin \frac{\chi_{1}}{2} \sin \frac{\chi_{2}}{2} \sin G \gamma\left(\pi-\frac{2 \pi}{m}\right)\right]
\end{aligned}
$$

Note that the vertical component of the stable-spin direction is independent of the orbit angle $\theta$. Only the horizontal and longitudinal components are dependent on the orbit angle.

In the two-detector case, the two solenoids are separated by one sixth of the ring, or $m=6$. The spin-tune is given by

$$
\begin{aligned}
\cos \pi \nu_{\mathrm{s}}= & \cos \frac{\chi_{1}}{2} \cos \frac{\chi_{2}}{2} \cos G \gamma \pi \\
& -\sin \frac{\chi_{1}}{2} \sin \frac{\chi_{2}}{2} \cos G \gamma\left(\frac{2 \pi}{3}\right)
\end{aligned}
$$

Here, the effective resonance strengths of the two partial snakes will be modulated by $G \gamma$. The imperfection resonance strengths of the three different lattices calculated from DEPOL [16] are plotted in Fig. 3. The two dashed lines represents the thresholds for a 99\% spin flip and $1 \%$ polarization loss based on the realistic RHIC ramp rate and Eq. (1) with $\chi=0$ (no partial snake). Between the two dashed lines, the resonance strengths are sufficiently strong to cause sizable polarization losses. As can be observed, the snake strength of the dual partial snake varies around the single partial snake case with a period of $|G \gamma|=6$. The polarization after each imperfection resonance can be calculated using the Froissart-Stora formula for each lattice. For example, Fig. 4 depicts the polarization after each imperfection resonance for a lattice with $\nu_{y}=30.224$. For the case with two partial snakes, the worst case scenario is assumed, where the partial snakes and imperfection spin resonance are out of phase. The results show that the two detector solenoids are sufficiently strong to achieve more than $99 \%$ polarization through each imperfection resonance.

For the one-detector solenoid case, the spin-tune and stable spin direction can be deduced from Eqs. (7)-(9) by letting $\chi_{2}=0$ and $m \rightarrow \infty$ :

$$
\cos \pi \nu_{\mathrm{s}}=\cos \frac{\chi_{1}}{2} \cos G \gamma \pi
$$

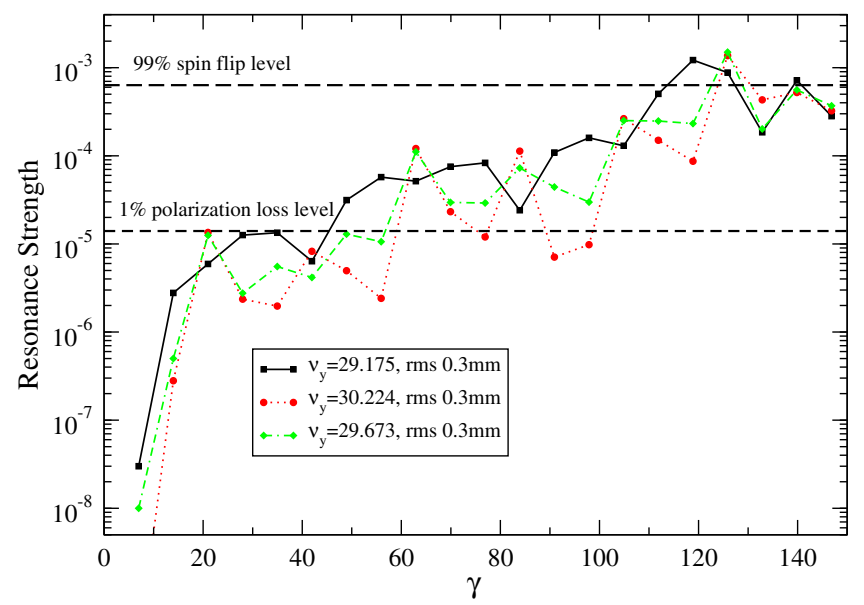

FIG. 3. The imperfection resonance strength with three ion-ring lattices. The partial snake resonance strengths for single and dual detector solenoids are shown. From Eq. (4), the partial snake strength decreases as the energy increases for a constant solenoid field. The lines between points are only drawn to guide the eye. The resonance strengths between the two straight lines cause a polarization loss of over $1 \%$ with the nominal ramp rate.

$$
\begin{aligned}
& \cos \alpha_{1}=\frac{-1}{\sin \pi \nu_{\mathrm{s}}}\left[\sin \frac{\chi_{1}}{2} \sin G \gamma(\pi-\theta)\right], \\
& \cos \alpha_{2}=\frac{1}{\sin \pi \nu_{\mathrm{s}}}\left[\sin \frac{\chi_{1}}{2} \cos G \gamma(\pi-\theta)\right], \\
& \cos \alpha_{3}=\frac{1}{\sin \pi \nu_{\mathrm{s}}}\left[\cos \frac{\chi_{1}}{2} \sin G \gamma \pi\right]
\end{aligned}
$$

It can be observed from Eq. (11) that with $\theta=0$ (at the detector location) and $|G \gamma|=$ integer, the spin is in the longitudinal direction. The resonance strength of detector 1

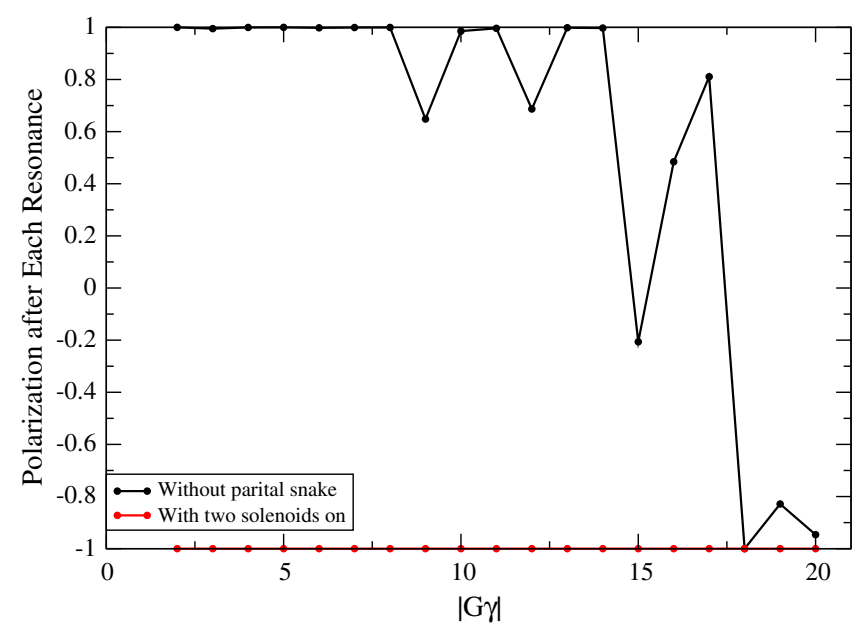

FIG. 4. The polarization after each imperfection resonance for a lattice with $\nu_{y}=30.224$. The polarization is preserved with the two solenoids. Each data point represents the polarization value after crossing an imperfection resonance. The lines between points are only drawn to guide the eye. 
is plotted in Fig. 3. With detector 1 running alone, this acts as one partial snake, and is sufficiently strong. With detector 2 running alone, additional solenoid fields are required at the detector 2 location to preserve the polarization.

Partial snakes tilt the stable spin direction away from the vertical. With injection at $|G \gamma|=1.5$, the strengths of the two partial snakes are about $6 \%$ and $2 \%$, respectively. The vertical spin component is 0.995 out of 1 in the case with two partial snakes or just 6\% partial snake alone from Eqs. (9) and (11). This is a small tilt. In addition, the nonvertical stable spin direction introduces depolarizing resonances related to the horizontal tune, as the so-called horizontal intrinsic resonances [19]. However, these only have a strong enough resonance strength for partial snakes with a strength higher than $10 \%$. For the partial snake strengths considered in this study, the horizontal resonance effect is negligible.

\section{LONGITUDINAL POLARIZATION}

Physics experiments require polarization along the longitudinal direction at the detectors. For protons, this is achieved by using spin rotators to rotate the vertical spin into and out of the longitudinal direction in the interaction region (IR). In the BNL EIC ion ring, the stable-spin direction of the deuteron beam is nearly vertical except when $G \gamma$ is near an integer. In general, it is impossible to build spin rotators to rotate the spin from vertical to longitudinal, owing to the small $G$ value. However, at $G \gamma=$ integer, the stable-spin direction will precess in the horizontal plane. For certain energies, polarization is naturally in the longitudinal direction at the experimental IRs. For a single-detector operation scenario, as shown in Eq. (11), the vertical $\left(\cos \alpha_{3}\right)$ and horizontal $\left(\cos \alpha_{1}\right)$ components are both zero when $G \gamma$ is an integer. Therefore, experiments with longitudinal spin are possible at the discrete energies with $|G \gamma|=$ integer, or approximately every $6.58 \mathrm{GeV}$.

The most likely operation scenario for the BNL EIC is to operate with two detectors. The two-detector scenario is more complicated. At the first partial snake with $m=6$ and $\theta=0$, the stable spin direction is

$$
\begin{aligned}
\cos \alpha_{1}= & \frac{-1}{\sin \pi \nu_{\mathrm{s}}}\left[\cos \frac{\chi_{1}}{2} \sin \frac{\chi_{2}}{2} \sin G \gamma\left(\frac{4 \pi}{3}\right)\right. \\
& \left.+\sin \frac{\chi_{1}}{2} \cos \frac{\chi_{2}}{2} \sin G \gamma \pi\right], \\
\cos \alpha_{2}= & \frac{1}{\sin \pi \nu_{\mathrm{s}}}\left[\cos \frac{\chi_{1}}{2} \sin \frac{\chi_{2}}{2} \cos G \gamma\left(\frac{4 \pi}{3}\right)\right. \\
& \left.+\sin \frac{\chi_{1}}{2} \cos \frac{\chi_{2}}{2} \cos G \gamma \pi\right], \\
\cos \alpha_{3}= & \frac{1}{\sin \pi \nu_{\mathrm{s}}}\left[\cos \frac{\chi_{1}}{2} \cos \frac{\chi_{2}}{2} \sin G \gamma \pi\right. \\
& \left.-\sin \frac{\chi_{1}}{2} \sin \frac{\chi_{2}}{2} \sin G \gamma \frac{2 \pi}{3}\right]
\end{aligned}
$$

Similarly, at the second partial snake with $m=6$ and $\theta=-2 \pi / 6$, the stable spin direction is

$$
\begin{aligned}
\cos \alpha_{1}= & \frac{-1}{\sin \pi \nu_{\mathrm{s}}}\left[\cos \frac{\chi_{1}}{2} \sin \frac{\chi_{2}}{2} \sin G \gamma\left(\frac{4 \pi}{3}\right)\right. \\
& \left.+\sin \frac{\chi_{1}}{2} \cos \frac{\chi_{2}}{2} \sin G \gamma \pi\right], \\
\cos \alpha_{2}= & \frac{1}{\sin \pi \nu_{\mathrm{s}}}\left[\cos \frac{\chi_{1}}{2} \sin \frac{\chi_{2}}{2} \cos G \gamma\left(\frac{4 \pi}{3}\right)\right. \\
& \left.+\sin \frac{\chi_{1}}{2} \cos \frac{\chi_{2}}{2} \cos G \gamma \pi\right], \\
\cos \alpha_{3}= & \frac{1}{\sin \pi \nu_{\mathrm{s}}}\left[\cos \frac{\chi_{1}}{2} \cos \frac{\chi_{2}}{2} \sin G \gamma \pi\right. \\
& \left.-\sin \frac{\chi_{1}}{2} \sin \frac{\chi_{2}}{2} \sin G \gamma \frac{2 \pi}{3}\right]
\end{aligned}
$$

It is clear that the horizontal and vertical components are zero when $G \gamma=3 n$, where $n$ is an integer. Therefore, experiments with longitudinal spin are possible at the discrete energies with $|G \gamma|=3 \times$ integer, or approximately every $19.74 \mathrm{GeV}$.

In addition, to maintain longitudinal polarization using a solenoid, the spin-tune spread must be considered. For a synchrotron without a full or strong partial snake, the spintune spread is given by [20]

$$
\Delta \nu_{\mathrm{s}}=\beta^{2} G \gamma \frac{d p}{p}
$$

where $\beta$ is the velocity relative to the speed of light. From experience of unpolarized deuteron operation in RHIC, the full width of the momentum spread $\frac{d p}{p}$ is approximately $1.28 \times 10^{-3}$ at injection and $0.43 \times 10^{-3}$ at $|G \gamma|=18$. The spin-tune spread is to the order of 0.0077 for the highest possible energy with $|G \gamma|=3 n$ at $|G \gamma|=18$. The induced resonance strength at an integer must be larger than the spin-tune spread, so that all particles are covered by the resonance. The resonance strength of a partial snake is to the order of 0.00225. By itself, this is not sufficient to maintain a longitudinal direction for all particles at $|G \gamma|=18$. One solution is to add orbit errors to enhance the imperfection resonance. This can be achieved using a harmonic bump, to bring the rms orbit to the order of 4-6 $\mathrm{mm}$ at the given energy.

For lower energies, such as $|G \gamma|=3,6$, the resonance strength of the solenoid partial snake is sufficiently strong. At $|G \gamma|=18$, a harmonic orbit bump with both sine and cosine terms can be used to generate a large resonance strength. A strength of 0.025 can be achieved with a rms orbit of $6 \mathrm{~mm}$, and a strength of 0.02 can be reached with a rms orbit of $4.5 \mathrm{~mm}$. This harmonic orbit bump is only required after acceleration is completed, and thus the 
remainder of the resonances on the ramp are not affected. Similarly, at $|G \gamma|=15$ a resonance strength of 0.021 can be generated by a rms orbit of $4.4 \mathrm{~mm}$. This strength is enough for the spin-tune spread of 0.007 at this energy. In summary, a longitudinal polarization can be achieved at certain $|G \gamma|$ values by introducing additional harmonic orbits to correct for the expected spin-tune spread owing to the momentum spread.

\section{OVERCOMING INTRINSIC SPIN RESONANCES}

The intrinsic resonance strength can also be calculated from DEPOL [16]. Based on previous unpolarized deuteron beam operations in RHIC, a $2 \mu \mathrm{m}$ normalized rms beam emittance is expected. Figure 5 depicts the resonance strengths with a rms emittance of $2 \mu \mathrm{m}$ for a few lattices. The three-fold symmetry means that the stronger resonances occur at $G \gamma=3 n \pm \nu_{y}$. However, owing to the slow ramp rate, other resonances can also cause polarization losses, which were not included in previous studies. The strongest resonance is at $G \gamma=12-\nu_{y}$, where the strength is approximately $3.5 \times 10^{-3}$ for a $2 \mu \mathrm{m}$ emittance beam. This resonance can fully flip the spin with the nominal ramp rate.

There are several possible methods of overcoming intrinsic resonances with strengths between the two dashed lines in Fig. 5. An AC dipole has been utilized in the AGS before dual partial snakes were employed [9]. The idea is to generate an artificial resonance strength near the existing intrinsic resonance to overpower it. However, for some relatively weak resonances, the required betatron oscillation is beyond the available physical aperture. The betatron

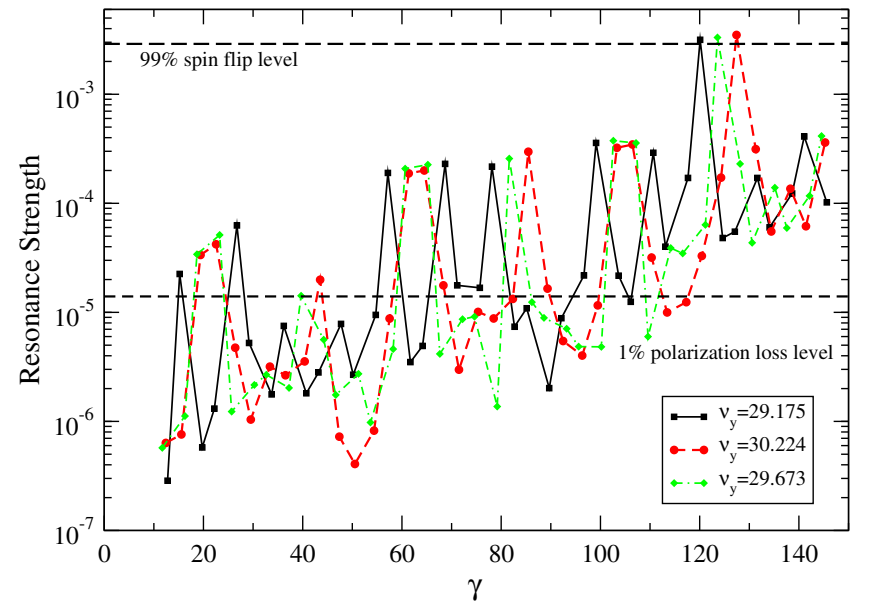

FIG. 5. The intrinsic resonances for a few lattices calculated by DEPOL. The two dashed lines are calculated with a Gaussian beam of $2 \mu \mathrm{m}$ rms emittance. The resonance strengths between the two lines cause polarization losses of over $1 \%$ with the nominal ramp rate. The lines between points are only drawn to guide the eye. oscillation is limited to a $5 \sigma$-rms beam size by aperture. This amplitude is not sufficient to overcome all intrinsic resonances in the energy range.

On the other hand, a vertical tune jump can be utilized to provide a fast resonance crossing speed. From experience with the AGS, it is not necessary to jump the betatron tune in one turn to maintain the polarization over a strong intrinsic resonance. It has been demonstrated that a vertical tune jump of 0.2 over 10 orbit turns is effective, and the emittance growth in this case is considerably smaller [7]. A benign horizontal tune jump scheme in the AGS has also been implemented in recent operations to overcome the horizontal intrinsic resonances [19] induced by the partial Siberian snakes [6]. A tune jump of 0.04 over 37 turns did not lead to more than $10 \%$ emittance growth. Note that although the AGS horizontal tune jump system was intended for the horizontal plane, it also induced a vertical tune jump of approximately 0.02 . As the vertical betatron tune is close to an integer ( 0.98 or closer), the emittance growth is in the vertical, but not visible in the horizontal. In the case of the BNL EIC, where the working point is away from an integer, it is expected that the emittance growth would be negligible.

The final polarization after crossing an isolated depolarizing resonance is given by the Froissart-Stora formula [18]. When integrated over a Gaussian beam, this yields [19]

$$
\frac{P_{\mathrm{f}}}{P_{\mathrm{i}}}=\frac{1-\frac{\pi|\epsilon|^{2}}{\alpha}}{1+\frac{\pi|\epsilon|^{2}}{\alpha}}
$$

In the case of a tune jump, the resonance crossing rate is

$$
\alpha=\frac{d(G \gamma)}{d \theta} \pm \frac{d \nu_{y}}{d \theta} .
$$

To overcome the resonance strength shown in Fig. 5 and achieve over 99\% polarization preservation for each resonance, the required resonance crossing speed should be approximately $9.5 \times 10^{-5}$, or about 800 times the value of the regular ramp rate. Next, the required tunejump amplitude needs to be determined. As shown in Eq. (14), the spin-tune spread at the highest intrinsic resonance $\left(|G \gamma|=-9+\nu_{\mathrm{y}}=20.673\right)$ is approximately 0.0264. For the tune jump to be effective for all particles, the tune-jump amplitude must be larger than the spin-tune spread of 0.0264. With nonzero chromaticity and momentum spread, the resonance conditions should be rewritten as

$$
\begin{aligned}
& G \gamma=N-\left(\nu_{y} \pm \xi_{y} d p / p\right), \\
& G \gamma=N+\left(\nu_{y} \pm \xi_{y} d p / p\right),
\end{aligned}
$$

where $\xi_{y}$ is the vertical chromaticity, and $\pm d p / p$ gives the up and down boundaries of the momentum distribution. 
In RHIC operation, the majority of the ramp is above the transition, and the vertical chromaticity on the ramp above the transition is approximately 2. The spread of the resonance line owing to the nonzero chromaticity will add or subtract an additional $2 d p / p \sim 0.00256$ in the tune jump amplitude, depending on whether the $N-\nu_{y}$ or $N+\nu_{y}$ resonance is crossed. A tune-jump amplitude of 0.03 takes into account this effect, and more importantly the spin-tune spread. With $\alpha=9.5 \times 10^{-5}$ and a tune-jump amplitude of 0.03 , the required number of turns is 50 . This is a smaller tune jump over more turns in comparison to the AGS horizontal tune jump. With the vertical tune further away from an integer than in the AGS case, the emittance growth is not an issue. An example of the tune-jump scheme is illustrated in Fig. 6. To obtain full benefit, all particles in the beam have to cross the resonance during the tune jump. The case shown in Fig. 6 represent the scenario in which a nonzero chromaticity adds an additional 0.00256 to the tune-jump amplitude. This shows that particles over the whole beam benefit from the tune jump of 0.03 .

The required quadrupole strength and power supply can be estimated. The intrinsic resonance with the highest rigidity is at $|G \gamma|=-9+\nu_{\mathrm{y}}=20.673$ or $\gamma=135.6$, where $B \rho=904 \mathrm{Tm}$. Considering the three-fold symmetry of

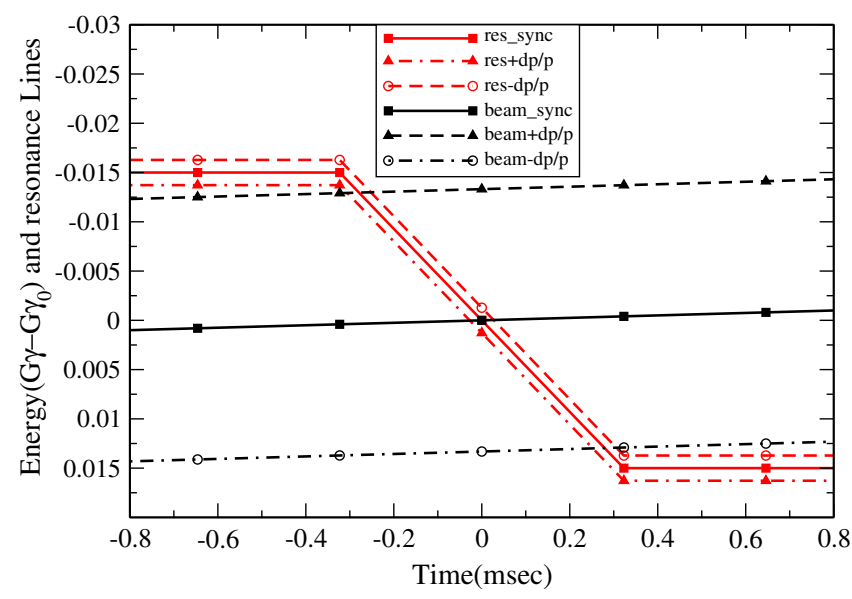

FIG. 6. Sketch of a resonance crossing with a tune jump for the highest resonance near $G \gamma=-20.673$, with a fractional vertical tune of 0.673 . The horizontal axis represents the relative time to the resonance crossing, and the vertical axis represents the energy relative to the synchronous particle at the jump time in units of $G \gamma$. The revolution frequency of RHIC varies between $77.8 \mathrm{kHz}$ and $78.2 \mathrm{kHz}$ for deuteron beam on the ramp. Note that the vertical axis is reversed as the $G$ value is negative. The solid line represents a synchronous particle, and the two broken lines represent the boundary particles at the positive and negative momentum-spread boundaries of the whole beam. The resonance lines (red) are given by Eq. (17) for positive and negative momentum spreads. The momentum half-width of $0.64 \times 10^{-3}$ is adopted, which is measured for an unpolarized deuteron. A chromaticity of 2 is utilized for this plot.
RHIC ion ring, three quadrupoles are used. To correct this resonance, a tune jump of $\Delta \nu_{\mathrm{y}}=0.01$ in 50 turns is required for each of the three quadrupoles. The required quadrupole strength is

$$
B^{\prime} l=\frac{4 \pi \Delta \nu_{\mathrm{y}}}{\beta} B \rho=2.27 T,
$$

where $\beta=50 \mathrm{~m}, \Delta \nu_{\mathrm{y}}=0.01$, and $l=0.8 \mathrm{~m}$ for each quadrupole. This yields $B^{\prime}=2.84 \mathrm{~T} / \mathrm{m}$. For the beam pipe radius $R=0.04 \mathrm{~m}$, the required current becomes

$$
N I=\frac{B^{\prime} R^{2}}{2 \mu_{0}}=1809 \mathrm{~A}-\text { turns. }
$$

Using a two-turn coil gives a required current of 905 A. This is similar to the design of the AGS horizontal tunejump system. The inductance for such a quadrupole is $57 \mu \mathrm{H}$, based on the standard inductance formula.

In RHIC, 50 turns require approximately $640 \mu \mathrm{s}$. The required voltage is

$$
V=L \frac{d I}{d t} \approx 81 V
$$

where $L=57 \mu \mathrm{H}$ and $640 \mu$ s are used. Thus a tune-jump system consisting of three quads and three power supplies of $1000 \mathrm{~A}$ and $100 \mathrm{~V}$, respectively, should be effective. This represents a modest tune-jump system compared to the two utilized in the AGS: the AGS vertical tune-jump system from the 1980s required $2250 \mathrm{~A}$ and $15 \mathrm{kV}$, with a rise time of $1.6 \mu \mathrm{s}$ [4], and the AGS horizontal tune-jump system currently in use requires $1500 \mathrm{~A}$ and $750 \mathrm{~V}$ [21-22].

The polarizations after each intrinsic resonance are illustrated in Fig. 7, where the results of the nominal ramp

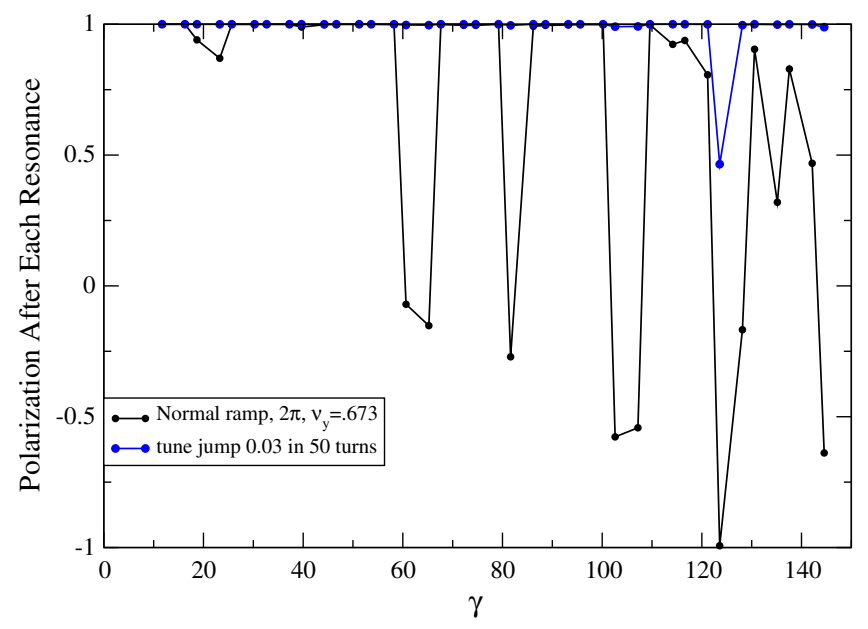

FIG. 7. The polarization after each intrinsic resonance with a $2 \mu \mathrm{m}$ normalized rms emittance. Each data point represents the polarization value after crossing an intrinsic resonance. The lines between points are only drawn to guide the eye. 
with and without a tune-jump system are presented. The tune-jump system can correct all the intrinsic spin resonances except for $|G \gamma|=\nu_{y}-12$, which will induce a full spin flip at the nominal ramp, without the need for the tunejump quadrupoles. The overall efficiency for the tune-jump method plus a normal ramp across $|G \gamma|=\nu_{y}-12$ is $95 \%$. The lines between points are only drawn to guide the eye.

\section{OVERLAPPING RESONANCES AND SYNCHROTRON SIDEBANDS}

In this paper, we treated all resonances as isolated resonances, and Froissart-Stora formula is used to treat each resonance. A natural question is weather this treatment is valid. To treat two resonances next to each other as separated resonances, the distance between the two resonances $\delta$ should be much larger than the maximum of the two resonance strengths $\epsilon_{1}$ and $\epsilon_{2}$. The closest distance between intrinsic and imperfection resonances are for the case $\nu_{y}=0.175$ where the distance is 0.175 in the tune space. Since most of the resonances are less than or in the order of 0.001 , the ratio of the separation of the two resonances to the resonance strength is more than two orders of magnitude, which satisfies the condition for the isolated resonances: $\delta \gg \max \left(\epsilon_{1}, \epsilon_{2}\right)$.

In addition to the imperfection and intrinsic resonances, there are numerous synchrotron sideband resonances during the acceleration. The distances of these resonances are in distances of multiples of synchrotron tune, $\pm p \nu_{\text {syn }}$ from the parent ones, where $p$ is the sideband order [23]. The strength of each sideband is the product of the parent resonance strength $\epsilon$ and the Bessel function $J_{p}(x)$, where $x$ is the ratio of the spin-tune modulation depth $G \Delta \gamma$ over the synchrotron tune $\nu_{\text {syn }}$ [24]. The synchrotron tune for deuteron beam in RHIC along the ramp has been measured as $3 \times 10^{-3}$ at injection and $3 \times 10^{-4}$ at $100 \mathrm{GeV}$. On the ramp, the spin resonance strength of the solenoids reduces from 0.03 down to 0.0023 at top energy. In general, the spin-tune modulation depth $G \Delta \gamma$ should be smaller than the solenoidal partial snake strength so that resonances from solenoids overpower the parent resonance as well as the synchrotron sidebands for all particles with different synchrotron oscillation amplitudes. This is the case for the injection and middle range of the ramp. Near the top energy where $G \Delta \gamma=0.008$, the resonance strength from solenoid is only 0.0023 which is not enough to cover the expected spin-tune modulation depth 0.008 . As discussed in the end of Sec. IV, harmonic orbit bumps need to be added to generate a stronger resonance strength. As an example, for $G \gamma=18$, the orbit harmonic of $6 \mathrm{~mm}$ generates resonance strength of 0.025 , much larger than the spin-tune modulation depth of 0.008 . With the combination of solenoids and harmonic orbit bumps, the resonance strengths from them are much stronger than the strengths of the synchrotron sidebands. As a result, the resonances from them overpower the parent resonance as well as the synchrotron sidebands. For the intrinsic resonance case, the synchrotron motion effect has been considered and wider tune jump has been proposed. The 0.03 jump in tune space is much larger than the synchrotron tune $\left(10^{-3}\right.$ to $10^{-4}$ ).

\section{CONCLUSION}

The possibility of accelerating polarized deuterons in the BNL EIC has been explored in detail. The resonance strengths have been calculated for various RHIC lattices. Furthermore, several possible schemes to overcome these resonances have been analyzed. We found that the imperfection spin resonances can be overcome by using the detector solenoids as partial snakes. This has the additional benefit of ensuring longitudinal polarization at $|G \gamma|=3 \times$ integer, together with vertical orbit bumps. Intrinsic spin resonances can be handled using a modest tune-jump system. In summary, this paper describes the feasibility for polarized deuteron acceleration in BNL EIC. Adding a modest tune-jump system and using detector solenoids in combination with harmonic orbit bumps in the BNL EIC would likely preserve the deuteron beam polarization to the full energy. In the future design work of polarized deuteron in BNL EIC, front-to-end particle simulation is planned.

\section{ACKNOWLEDGMENTS}

This work was supported by Brookhaven Science Associates, LLC, under Contract No. DE-AC0298CH10886 with the U.S. Department of Energy.

[1] Y.S. Derbenev and A. M. Kondratenko, Radiative polarization: Obtaining, control, using, Part. Accel. 8, 115 (1978).

[2] I. Alekseev et al., Polarized proton collider at RHIC, Nucl. Instrum. Methods Phys. Res., Sect. A 499, 392 (2003).

[3] A. Accardi et al., Electron-Ion Collider: The next QCD frontier, Eur. Phys. J. A 52, 268 (2016).

[4] F. Z. Khiari et al., Acceleration of polarized protons to 22 $\mathrm{GeV} / \mathrm{c}$ and the measurement of spin-spin effects in $p \uparrow p \uparrow \rightarrow p+p$, Phys. Rev. D 39, 45 (1989).

[5] H. Huang et al., Preservation of Proton Polarization by a Partial Siberian Snake, Phys. Rev. Lett. 73, 2982 (1994).

[6] H. Huang et al., Overcoming Depolarizing Resonances with Dual Helical Partial Siberian Snakes, Phys. Rev. Lett. 99, 154801 (2007).

[7] T. Roser, Polarized proton beams, in Proceedings of the Particle Accelerator Conference, Dallas, TX, 1995 (IEEE, New York, 1995), p. 3154.

[8] H. Huang et al., Overcoming horizontal depolarizing resonances with multiple tune jumps, Phys. Rev. Accel. Beams 17, 081001 (2014). 
[9] M. Bai et al., Overcoming Intrinsic Spin Resonances with an rf Dipole, Phys. Rev. Lett. 80, 4673 (1998).

[10] H. Huang et al., Polarized proton experiment in the AGS with a partial snake, in Proceedings of the 12th International Symposium on High Energy Spin Physics, Amsterdam, Netherlands (World Scientific, Singapore, 1996), pp. 528.

[11] H. Huang et al., Overcoming an intrinsic depolarizing resonance with a partial Siberian snake, Phys. Rev. Accel. Beams 7, 071001 (2004).

[12] S. Y. Lee and L. G. Ratner, Feasibility of a polarized deuteron beam in the AGS and RHIC, Nucl. Instrum. Methods Phys. Res., Sect. A 306, 51 (1991).

[13] E. D. Courant, Polarized neutrons in RHIC, BNL RHIC Tech Note 142, 1998 (https://technotes.bnl.gov/PDF? publicationId=36076).

[14] W. W. MacKay, Prospects for acceleration of deuterons and helions, Prospects for acceleration of deuterons and helions, AIP Conf. Proc. 980, 191 (2008).

[15] The energy referred in this paper is the energy per nucleon.

[16] E. D. Courant and R. Ruth, Polarized proton beams, Report No. BNL 51270, 1980, https://technotes.bnl.gov/PDF? publicationId $=36076$.
[17] https://zgoubi.sourceforge.io/ZGOUBIDOCS/Zgoubi.pdf; https://www.osti.gov/scitech/biblio/1062013-zgoubiusers-guide.

[18] M. Froissart and R. Stora, Depolarisation d'un faisceau de protons polarises dans un synchrotron, Nucl. Instrum. Methods 7, 297 (1960).

[19] F. Lin et al., Exploration of horizontal intrinsic spin resonances with two partial Siberian snakes, Phys. Rev. Accel. Beams 10, 044001 (2007).

[20] S. Y. Lee, Spin Dynamics and Snakes in Synchrotrons (World Scientific, Singapore, 1997), p. 53.

[21] J. L. Mi et al., AGS tune jump power supply design and test, in Proceedings of the 24th Particle Accelerator Conference, PAC-2011, New York, 2011 (IEEE, New York, 2011), p. 1298.

[22] J. W. Glenn et al., AGS fast spin resonance jump, magnets and power supplies, in Proceedings of the 23rd Particle Accelerator Conference, Vancouver, Canada, 2009 (IEEE, Piscataway, NJ, 2009), p. 1699.

[23] B. W. Montague, Polarized beams in high energy storage rings, Phys. Rep. 113, 1 (1984).

[24] S. Y. Lee and M. Berglund, Overlapping spin synchrotron sideband resonances, Phys. Rev. E 54, 806 (1996). 